Pacific

Journal of

Mathematics

UNITARY REPRESENTATIONS OF CLASSICAL LIE GROUPS OF EQUAL RANK WITH NONZERO DIRAC COHOMOLOGY

Hou Zixin, Liang Ke, and Zhu Fuhai

Volume 214 No. 2

April 2004 


\title{
UNITARY REPRESENTATIONS OF CLASSICAL LIE GROUPS OF EQUAL RANK WITH NONZERO DIRAC COHOMOLOGY
}

\author{
Hou Zixin, Liang Ke, And Zhu Fuhai
}

\begin{abstract}
In this paper, we consider unitary representations of classical groups of equal rank $(\operatorname{rank} G=\operatorname{rank} K)$ except type $C I$ with regular lambda-lowest $K$-type and get the necessary and sufficient condition such that those unitary representations considered have nonzero Dirac cohomology.
\end{abstract}

\section{Introduction.}

In the past twenty years, people are interested in unitary representations with nonzero cohomologies, that is, $(\mathfrak{g}, K)$-cohomology and Dirac cohomology. The former was studied by Vogan and Zuckerman in [10]. Since every representation with nonzero $(\mathfrak{g}, K)$-cohomology has nonzero Dirac cohomology, maybe it is this fact that motivates people to pay more attention to Dirac cohomology.

In 1997, Vogan explained a conjecture on Dirac cohomology at MIT Lie groups seminar. The conjecture can be stated as follows: Let $G$ be a connected semisimple Lie group with Lie algebra $\mathfrak{g}_{0}$ and let $K$ be the maximal compact subgroup of $G$ corresponding to the Cartan involution $\theta$. Suppose $X$ is an irreducible unitarizable $(\mathfrak{g}, K)$-module and $(\gamma, S)$ is a space of spinors for $\mathfrak{p}_{0}$. Here $\mathfrak{g}_{0}=\mathfrak{k}_{0}+\mathfrak{p}_{0}$ is the Cartan decomposition of $\mathfrak{g}_{0}$. Let $x_{1}, \ldots, x_{n}$ be an orthonormal basis of $\mathfrak{p}_{0}$, then the Dirac operator $D=\sum \pi\left(x_{i}\right) \otimes \gamma\left(x_{i}\right)$ acts on $X \otimes S$. Vogan's conjecture says that if $D$ has nonzero Dirac cohomology, which by definition is just $\operatorname{Ker} D$, then the infinitesimal character of $X$ can be expressed in terms of the highest weight of a $K$-type of $X$.

The conjecture was proved by Huang and Pandžić [2]. Furthermore, they get that an irreducible unitarizable $(\mathfrak{g}, K)$-module $X$ has nonzero Dirac cohomology, say $\gamma \subseteq \operatorname{Ker} D$, if and only if the infinitesimal character $\Lambda$ of $X$ is given by $\gamma+\rho_{c}$. To be precise, $\gamma$ has highest weight $\omega\left(\mu-\rho_{n}\right)$, where $\mu$ is a $K$-type of $X, \omega \in W(K)$ such that $\omega\left(\mu-\rho_{n}\right)$ is dominant and $\Lambda=\omega\left(\mu-\rho_{n}\right)+\rho_{c}$. One could ask: For what kinds of $K$-types does the expression $\left\|\omega\left(\mu-\rho_{n}\right)+\rho_{c}\right\|$ reach the minimum? For what cases is $\mu$ a lambda-lowest $K$-type of $X$ when $\omega\left(\mu-\rho_{n}\right) \subset \operatorname{Ker} D$ ? 
In this paper, we will answer the above problems partially. We study the representations of classical group $G$ of equal rank except type $C I$, with regular lambda-lowest $K$-type. First we recall the definition of $\theta$-stable data.

Definition 1.1 (Vogan [8], Definition 6.5.1). A set of $\theta$-stable data for $G$ is a quadruple $(\mathfrak{q}, H, \delta, \nu)$, such that:

a) $\mathfrak{q}=\mathfrak{l}+\mathfrak{u}$ is a $\theta$-stable parabolic subalgebra of $\mathfrak{g}$. Let $L$ be the normalizer of $\mathfrak{q}$ in $G$.

b) $L$ is quasisplit, and $H=T A \subseteq L$ is a maximally split $\theta$-stable Cartan subgroup of $L$.

c) $\delta \in \hat{T}$ is fine with respect to $L$.

d) $\nu \in \hat{A}$.

e) Write $\lambda^{L} \in \mathfrak{t}^{*}$ for the differential of $\delta$, and $\lambda=\lambda^{L}+\rho(\Delta(\mathfrak{u}, \mathfrak{t})) \in \mathfrak{t}^{*} \subseteq \mathfrak{h}^{*}$. Then $\lambda$ is strictly dominant for $\Delta(\mathfrak{u}, \mathfrak{h})$.

There is a surjective map from the set of equivalence classes of irreducible $(\mathfrak{g}, K)$-module to $K$ conjugacy classes of set of $\theta$-stable data for $G$ ([8], Corollary 6.5.13). And following Vogan's method ([8], Chapter 5), one can construct $\theta$-stable data from any given irreducible $(\mathfrak{g}, K)$-module $X$.

Now we can state our main theorem.

Theorem 1.2. Let $X$ be an irreducible $(\mathfrak{g}, K)$-module with regular lambdalowest $K$-type $\mu$. Then $X$ is unitary and has nonzero Dirac cohomology if and only if the parameter $\nu$ in the $\theta$-stable data $(\mathfrak{q}, H, \delta, \nu)$ corresponding to $X$ is just $\frac{1}{2} \sum_{\beta_{i} \in \Gamma_{1}} \beta_{i}$ under $G$-conjugation. Here, $\Gamma_{1}$ is a set of roots defined by the lambda-lowest $K$-type $\mu$ during the construction of $\theta$-stable data (see Section 3.1 for details).

The paper is organized as follows: We first collected some notations and results on Dirac operator and Dirac cohomology in Section 2. Then we followed Vogan's method to construct $\theta$-stable data $(\mathfrak{q}, H, \delta, \nu)$ for corresponding $(\mathfrak{g}, K)$-module $X$. Actually, we found that the quasisplit subgroup $L$ is simple enough under our assumption. Locally $L$ is a product of copies of $S L(2, \mathbb{R})$ and Euclidean space. In Section 4, we find out that if a lambdalowest $K$-type $\mu$ of $X$ is regular, then $\mu-\rho_{n}$ is dominant (Proposition 4.2) and $\left\|\mu-\rho_{n}+\rho_{c}\right\| \leq\left\|\omega\left(\mu^{\prime}-\rho_{n}^{\prime}\right)+\rho_{c}\right\|$. Then $X$ has nonzero Dirac cohomology only if $\|\Lambda\|=\left\|\mu-\rho_{n}+\rho_{c}\right\|$. Fortunately, in this case, $\Lambda$ is dominant. Then Vogan's result, Theorem $1.3[\mathbf{9}]$, implies that $X$ is unitary, hence $X$ has nonzero Dirac cohomology by Huang and Pandžić's result (Proposition 2.4) since $\|\Lambda\|=\left\|\mu-\rho_{n}+\rho_{c}\right\|$. Thus we get the main theorem.

\section{Preliminary.}

Let $G$ be a real semisimple group with Lie algebra $\mathfrak{g}_{0}$ and let $K$ be the maximal compact subgroup of $G$ corresponding to Cartan involution $\theta$. Let 
$\mathfrak{g}_{0}=\mathfrak{k}_{0} \oplus \mathfrak{p}_{0}$ be the corresponding Cartan decomposition of $\mathfrak{g}_{0}$. Fix a maximally compact Cartan subalgebra $\mathfrak{h}_{0}^{c}$ of $\mathfrak{g}_{0}$ with decomposition $\mathfrak{h}_{0}^{c}=\mathfrak{t}_{0}^{c}+\mathfrak{a}_{0}^{c}$. Denote by be $\mathfrak{g}, \mathfrak{k}, \mathfrak{p}, \mathfrak{h}^{c}, \mathfrak{t}^{c}$ and $\mathfrak{a}^{c}$ the complexifications of $\mathfrak{g}_{0}, \mathfrak{k}_{0}, \mathfrak{p}_{0}, \mathfrak{h}_{0}^{c}$, $\mathfrak{t}_{0}^{c}$ and $\mathfrak{a}_{0}^{c}$, respectively. Let $\Delta\left(\mathfrak{g}, \mathfrak{h}^{c}\right)$ be the root system of $\mathfrak{g}$ with respect to $\mathfrak{h}^{c}$. Fix a system of positive roots, $\Delta^{+}\left(\mathfrak{k}, \mathfrak{t}^{c}\right)$, for $\Delta\left(\mathfrak{k}, \mathfrak{t}^{c}\right)$ and choose a compatible system of positive roots, $\Delta^{+}\left(\mathfrak{g}, \mathfrak{h}^{c}\right)$, for $\Delta\left(\mathfrak{g}, \mathfrak{h}^{c}\right)$ with the set of simple roots $\Pi=\left\{\alpha_{1}, \ldots, \alpha_{l}\right\}$. Let $G_{0}$ be the identity component of $G$.

Definition 2.1 ([11]). Let $(\pi, X)$ be a $(\mathfrak{g}, K)$-module, set $S=S\left(\mathfrak{p}_{0}\right)$, a space of spinors of $\mathfrak{p}_{0}$. Let $x_{1}, \ldots, x_{n}$ be an orthonormal basis of $\mathfrak{p}_{0}$, then the Dirac operator

$$
D: X \bigotimes S \rightarrow X \bigotimes S
$$

is defined by

$$
D=\sum \pi\left(x_{i}\right) \otimes \gamma\left(x_{i}\right)
$$

which is a $K$-module homomorphism (sometime $\widetilde{K}$-module homomorphism, where $\widetilde{K}$ is a two-fold spin cover of $K$ ).

The Dirac cohomology of $X$ is defined by

$$
\text { Ker } D /(\operatorname{Ker} D \cap \operatorname{Im} D) \text {. }
$$

When $X$ is unitary, then Dirac operator is self-dual, then we can see that the Dirac cohomology of $X$ is just $\operatorname{Ker} D$.

The following result of Pathasarathy is well-known. It can be found in many papers.

Proposition 2.2 (Pathasarathy's Dirac Inequality). Let $X$ be an irreducible unitary $(\mathfrak{g}, K)$-module with infinitesimal character $\Lambda$. Fix a representation of $K$ occurring in $X$ of highest weight $\mu \in\left(\mathfrak{t}^{c}\right)^{*}$, and a positive root system $\Delta^{+}\left(\mathfrak{g}, \mathfrak{t}^{c}\right)$ for $\mathfrak{t}^{c}$ in $\mathfrak{g}$. Here $\mathfrak{t}^{c}$ is Cartan subalgebra of $\mathfrak{k}$. Write

$$
\rho_{c}=\rho\left(\Delta^{+}(\mathfrak{k}, \mathfrak{t})\right), \quad \rho_{n}=\rho\left(\Delta^{+}(\mathfrak{p}, \mathfrak{t})\right) .
$$

Fix an element $\omega \in W_{K}$ such that $\omega\left(\mu-\rho_{n}\right)$ is dominant for $\Delta^{+}(\mathfrak{k}, \mathfrak{t})$. Then

$$
\left(\omega\left(\mu-\rho_{n}\right)+\rho_{c}, \omega\left(\mu-\rho_{n}\right)+\rho_{c}\right) \geq(\Lambda, \Lambda) .
$$

The equality holds if and only if

$$
\Lambda=\omega\left(\mu-\rho_{n}\right)+\rho_{c} .
$$

The last assertion was obtained by Huang and Pandžić [2].

We also have another similar inequality.

Proposition 2.3. Let $V$ be an irreducible unitary $(\mathfrak{g}, K)$-module with Hermitian form $\langle$,$\rangle and infinitesimal character \Lambda$. Assume $\mu \in \hat{K}$ occurs in $V$. Then

$$
\|\Lambda\|^{2} \leq\left\|\mu+\rho_{c}\right\|^{2}-\left\|\rho_{c}\right\|^{2}+\|\rho\|^{2} .
$$


Proof. Let $\left\{x_{i}\right\}$ be a orthonormal basis of $\mathfrak{p}$ with respect to the Killing form. For $v \in V_{\mu}$ we have

$$
\left\langle x_{i} v, x_{i} v\right\rangle \geq 0 \Rightarrow\left\langle x_{i}^{2} v, v\right\rangle \leq 0 \Rightarrow\left\langle\left(c-c_{\mathfrak{k}}\right) v, v\right\rangle \leq 0 .
$$

Then the assertion follows easily.

In 1997, Vogan explained a conjecture on Dirac cohomology, which was proved by Huang and Pandžić [2]. We summarize their results as follows:

Proposition $2.4([2])$. Let $X$ be an irreducible unitarizable $(\mathfrak{g}, K)$-module with infinitesimal character $\Lambda$. Assume $X \otimes S$ contains a $\widetilde{K}$-type $\gamma$, i.e., $(X \otimes S)(\gamma) \neq 0$. Then the Dirac cohomology of $X$, Ker $D$, contains $(X \otimes$ $S)(\gamma)$ if and only if $\Lambda=\gamma+\rho_{c}$. Here $\gamma$ must be of the form $\omega\left(\mu-\rho_{n}\right)$ for some $\rho_{n}$ and $K$-type $\mu$ contained in $X$.

\section{Construction of $\theta$-stable data.}

In this section, we will make the following assumption:

Assumption 3.1. $G$ is a classical group with $\operatorname{rank} G=\operatorname{rank} K$, i.e., $\theta$ is an inner automorphism of $\mathfrak{g}_{0}$. Consequently $\mathfrak{h}_{0}^{c}=\mathfrak{t}_{0}^{c}$.

We will follow Vogan's method to construct $\theta$-stable data, actually, the main work is to determine the structure of the quasisplit subgroup $L$.

3.1. Basic facts. First, we rewrite Proposition 5.3.3 [8], since we assume $\operatorname{rank} G=\operatorname{rank} K$ and $\mathfrak{h}=\mathfrak{t}^{c}$.

Proposition $3.2([\mathbf{8}])$. For each $\Delta^{+}\left(\mathfrak{k}, \mathfrak{t}^{c}\right)$-dominant weight $\mu \in \hat{T}$, there is a unique element $\lambda \in\left(\mathfrak{t}^{c}\right)^{*}$ having the following properties: Fix a $\theta$-invariant positive root system $\Delta^{+}\left(\mathfrak{g}, \mathfrak{t}^{c}\right)$ for $\mathfrak{t}^{c}$ in $\mathfrak{g}$, making $\mu+2 \rho_{c}$ dominant; and write $\rho=\rho\left(\Delta^{+}\left(\mathfrak{g}, \mathfrak{t}^{c}\right)\right)$. Then $\lambda$ is dominant for $\Delta^{+}\left(\mathfrak{g}, \mathfrak{t}^{c}\right)$, and there is a set

$$
\Gamma=\left\{\beta_{1}, \ldots, \beta_{r}\right\} \subseteq \Delta^{+}\left(\mathfrak{g}, \mathfrak{t}^{c}\right)
$$

satisfying:

a) If we put

$$
\begin{aligned}
\widetilde{\lambda} & =\mu+2 \rho_{c}-\rho, \\
c_{i} & =-\left(\widetilde{\lambda}, \beta_{i}^{\vee}\right),
\end{aligned}
$$

then

$$
0 \leq c_{i} \leq 1
$$

and

$$
\lambda=\widetilde{\lambda}+\frac{1}{2} \sum c_{i} \beta_{i}
$$

b) If $(\lambda, \alpha)=0$ for $\alpha \in \Delta\left(\mathfrak{g}, \mathfrak{t}^{c}\right)$, then $\left(\alpha, \beta_{i}\right) \neq 0$ for some $i$.

c) The root $\beta_{1}$ is noncompact and simple. 
d) Write

$$
\mathfrak{g}^{1}=\mathfrak{g}^{\beta_{1}}, \mathfrak{h}^{1}=\left(\mathfrak{t}^{c}\right)^{\beta_{1}} .
$$

Then the positive system $\Delta^{+}\left(\mathfrak{g}, \mathfrak{t}^{c}\right) \cap \beta_{1}^{\perp}$ and its subset $\left\{\beta_{2}, \ldots, \beta_{r}\right\}$ for $\Delta\left(\mathfrak{g}^{1}, \mathfrak{h}^{1}\right)$ satisfy these same conditions for $\mathfrak{g}^{1}$ and the weight $\left.\mu\right|_{\mathfrak{g}^{1} \cap \mathfrak{t}^{c}}$.

e) If $c_{i} \neq 0$ and $c_{j}=0$, then $i<j$.

Under Assumption 3.1, we can get a stronger result.

Lemma 3.3. Let the notation be as above. Then

$$
c_{i}=0 \text { or } 1 \text {. }
$$

Proof. By Lemma 7.7.6 [1], we have

$$
\exp \left(2 \pi \sqrt{-1} \alpha^{\vee}\right)=e,
$$

where $e$ is the unit of $G$. Then $\left(\mu, \alpha^{\vee}\right)$ is an integer.

For convenience, we denote

$$
\begin{aligned}
& \Gamma_{1}=\left\{\beta_{i} \in \Gamma \mid c_{i}=1\right\}, \\
& \Gamma_{0}=\left\{\beta_{i} \in \Gamma \mid c_{i}=0\right\} .
\end{aligned}
$$

Let $\Pi$ be the system of simple roots of $\Delta^{+}\left(\mathfrak{g}, \mathfrak{t}^{c}\right)$. Set

$$
\begin{gathered}
\Sigma_{1}=\left\{\alpha \in \Pi \mid\left(\widetilde{\lambda}, \alpha^{\vee}\right)=-1\right\}, \\
\Sigma_{0}=\left\{\alpha \in \Pi \mid\left(\widetilde{\lambda}, \alpha^{\vee}\right)=0\right\} .
\end{gathered}
$$

Now we can define $\mathfrak{l}$ by

$$
\Delta\left(\mathfrak{l}, \mathfrak{t}^{c}\right)=\left\{\alpha \in \Delta\left(\mathfrak{g}, \mathfrak{t}^{c}\right) \mid\left(\lambda, \alpha^{\vee}\right)=0\right\} .
$$

Obviously, the Dynkin diagram of $\mathfrak{l}$ is a subdiagram of that of $\mathfrak{g}$ if we choose compatible orderings, i.e.,

$$
\Delta^{+}\left(\mathfrak{l}, \mathfrak{t}^{c}\right) \subseteq \Delta^{+}\left(\mathfrak{g}, \mathfrak{t}^{c}\right) .
$$

Denote by $\Pi_{\mathfrak{l}}$ the system of simple roots of $\mathfrak{l}$.

First we establish some lemmas.

Lemma 3.4. Let $\alpha$ and $\beta$ be adjacent simple roots of the same length. Then

$$
\left(\widetilde{\lambda},(\alpha+\beta)^{\vee}\right) \geq 0 .
$$

Proof. If both $\alpha$ and $\beta$ are compact or noncompact, then $\alpha+\beta$ is compact, so

$$
\left(\widetilde{\lambda},(\alpha+\beta)^{\vee}\right) \geq\left(\mu,(\alpha+\beta)^{\vee}\right) \geq 0 .
$$

Thus we can assume $\alpha$ is compact and $\beta$ is noncompact. Then

$$
\left(\widetilde{\lambda}, \beta^{\vee}\right) \geq-1
$$

and

$$
\left(\widetilde{\lambda}, \alpha^{\vee}\right)=\left(\mu, \alpha^{\vee}\right)+1 \text {. }
$$


So

$$
\left(\widetilde{\lambda},(\alpha+\beta)^{\vee}\right) \geq\left(\mu, \alpha^{\vee}\right) \geq 0 .
$$

Lemma 3.5. Let $\alpha, \beta$ and $\alpha+\beta \in \Delta\left(\mathfrak{g}, \mathfrak{t}^{c}\right)$. If $\left(\widetilde{\lambda}, \alpha^{\vee}\right) \geq 0$ and $\left(\widetilde{\lambda}, \beta^{\vee}\right) \geq 0$, then

$$
\left(\widetilde{\lambda},(\alpha+\beta)^{\vee}\right) \geq 0 .
$$

Proof. $(\alpha+\beta)^{\vee}=a \alpha^{\vee}+b \beta^{\vee}$, where $a$ and $b$ are positive.

Lemma 3.6. Assume $\mu$ is regular, i.e., $\left(\mu, \gamma^{\vee}\right) \geq 1$, for all $\gamma \in \Delta^{+}\left(\mathfrak{k}, \mathfrak{t}^{c}\right)$. Let $\alpha$ and $\beta$ be adjacent simple roots. If $(\alpha, \alpha)=2(\beta, \beta)$, then

$$
\left(\widetilde{\lambda},(\alpha+\beta)^{\vee}\right) \geq 0 .
$$

If $\alpha$ and $\beta$ have the same length, then

$$
\left(\widetilde{\lambda},(\alpha+\beta)^{\vee}\right) \geq 1 .
$$

Proof. Only the first assertion needs to prove. We treat it case by case.

Case I. Both $\alpha$ and $\beta$ are noncompact.

$$
\left(\widetilde{\lambda},(\alpha+\beta)^{\vee}\right)=\left(\mu,(\alpha+\beta)^{\vee}\right)+2-\left(\rho, 2 \alpha^{\vee}+\beta^{\vee}\right) \geq 1+2-3 \geq 0 .
$$

Case II. $\alpha$ is compact while $\beta$ is noncompact.

$$
\left(\widetilde{\lambda},(\alpha+\beta)^{\vee}\right)=\left(\widetilde{\lambda}, 2 \alpha^{\vee}+\beta^{\vee}\right) \geq 4-1 \geq 3 .
$$

Case III. $\alpha$ is noncompact while $\beta$ is compact.

$$
\left(\widetilde{\lambda},(\alpha+\beta)^{\vee}\right)=\left(\widetilde{\lambda}, 2 \alpha^{\vee}+\beta^{\vee}\right) \geq-2+2 \geq 0 .
$$

Corollary 3.7. Let $\alpha \in \Sigma_{1}, \beta \in \Sigma_{1} \cup \Sigma_{0}$. Then $(\alpha, \beta)=0$ for types AIII and $D_{l}$. If $\mu$ is regular for $\Delta^{+}\left(\mathfrak{k}, \mathfrak{t}^{c}\right)$, then it is true for any type.

Lemma 3.8. Assume $\mu$ is regular for $\Delta\left(\mathfrak{k}, \mathfrak{t}^{c}\right)$ and $\Gamma$ consists of simple roots of $\Delta\left(\mathfrak{g}, \mathfrak{t}^{c}\right)$. Then the simple roots of $\mathfrak{l}$ are noncompact.

Proof. If $\alpha \in \Pi$ is compact, then

$$
\left(\lambda, \alpha^{\vee}\right)=\left(\widetilde{\lambda}, \alpha^{\vee}\right)+\frac{1}{2} \sum c_{i}\left(\beta_{i}, \alpha^{\vee}\right) \geq 2-\frac{3}{2}>0 .
$$

The first inequality holds because $\alpha$ is adjacent to at most three simple roots of the same length or two simple roots of different length. So $\alpha \notin \Pi_{\mathfrak{l}}$. 
3.2. Main theorem. Now we can study the structure of $\mathfrak{l}$. Our purpose is to prove the following theorem:

Theorem 3.9. Let $\mu$ be a $K$-type of a $(\mathfrak{g}, K)$-module. Assume $\mu$ is regular for $\Delta\left(\mathfrak{k}, \mathfrak{t}^{c}\right)$. Then:

1) For types $A I I I, C I I$ and $D_{l}$, the Dynkin diagram of $\mathfrak{l}$ is discrete.

2) For types $B_{l}$ and $C I$, the Dynkin diagram of $\mathfrak{l}$ is either discrete or of the form

$$
\underbrace{A_{1} \times \cdots \times A_{1}}_{r-2} \times B_{2} .
$$

3) For type $B_{l}$. If $\mu$ is regular for $\Delta\left(\mathfrak{g}, \mathfrak{t}^{c}\right)$, then the Dynkin diagram of $\mathfrak{l}$ is discrete.

Let's deal with the problem case by case.

3.2.1. Type $A I I I$. In this subsection, we assume that the Lie algebra $\mathfrak{g}_{0}$ is of type $A I I I$.

\section{Proposition 3.10.}

1) Let $\alpha \in \Delta^{+}\left(\mathfrak{g}, \mathfrak{t}^{c}\right)$. If $(\alpha, \beta)=0$ for any $\beta \in \Sigma_{1}$, then

$$
\left(\widetilde{\lambda}, \alpha^{\vee}\right) \geq 0
$$

2) Those $\beta_{i}$ in Proposition 3.2 can be chosen to be simple.

3) If $\mu$ is regular for $\Delta\left(\mathfrak{k}, \mathfrak{t}^{c}\right)$, then

$$
\Pi_{\mathfrak{r}}=\Gamma \text {. }
$$

Proof.

1) Let $\alpha=\alpha_{i}+\cdots+\alpha_{k}$. If $\alpha$ is not adjacent to any $\beta \in \Sigma_{1}$, then $\alpha_{i}$, $\alpha_{k} \notin \Sigma_{1}$, so $\left(\widetilde{\lambda}, \alpha^{\vee}\right) \geq 0$ by Lemma 3.4 .

2) Choose a maximal subset $\Sigma_{0}^{\prime}$ of $\Sigma_{0}$ such that the elements of $\Sigma_{0}^{\prime}$ are orthogonal to each other. Then we claim that the set $\Gamma=\Sigma_{1} \cup \Sigma_{0}^{\prime}$ satisfies the condition of Proposition 3.2.

Firstly, we choose $\Gamma_{1}$ containing $\Sigma_{1}$. By 1) we have

$$
\Gamma_{1}=\Sigma_{1}
$$

Secondly, we choose $\Gamma_{0}$ containing $\Sigma_{0}^{\prime}$. If $\alpha=\alpha_{i}+\cdots+\alpha_{k}$ is orthogonal to $\Sigma_{1} \cup \Sigma_{0}^{\prime}$ and

$$
\left(\widetilde{\lambda}, \alpha^{\vee}\right)=0,
$$

then $\alpha_{i}, \alpha_{k} \notin \Sigma_{1} \cup \Sigma_{0}^{\prime}$. We claim that $\alpha_{i}, \ldots, \alpha_{k} \in \Sigma_{0}$. By Lemma 3.4, we have $\left(\tilde{\lambda},\left(\alpha_{i+1}+\cdots+\alpha_{k}\right)^{\vee}\right) \geq 0$. The equality holds and $\alpha_{i} \in \Sigma_{0}$ by Equation (1). Furthermore, for the same reason we have $\left(\widetilde{\lambda},\left(\alpha_{i}+\alpha_{i+1}\right)^{\vee}\right)=$ 0 , that is $\alpha_{i+1} \in \Sigma_{0}$. Then our claim follows. But one can easily see that the claim contradicts the fact that $\Sigma_{0}^{\prime}$ is maximal. 
3) Obviously, $\Gamma \subseteq \Pi_{\mathfrak{r}}$. Let $\alpha \in \Pi_{\mathfrak{r}} \backslash \Gamma$. By Lemma 3.8, $\alpha$ is noncompact. Then $\alpha$ must be adjacent to some $\beta \in \Gamma$, so $\left(\widetilde{\lambda},(\alpha+\beta)^{\vee}\right) \geq 1$. Hence $\left(\lambda, \alpha^{\vee}\right)>0$. Contradiction.

Corollary 3.11. If $\mu$ is regular for $\Delta\left(\mathfrak{g}, \mathfrak{t}^{c}\right)$, then $\widetilde{\lambda}$ is strictly dominant for $\Delta(\mathfrak{u})$, that is,

$$
\left(\widetilde{\lambda}, \alpha^{\vee}\right)>0
$$

for any $\alpha \in \Delta(\mathfrak{u})$.

Proof. Just follow the proof of the above proposition.

\subsubsection{Types $B_{l}$ and $C_{l}$.}

Proposition 3.12. Assume $\mu$ is regular for $\Delta\left(\mathfrak{k}, \mathfrak{t}^{c}\right)$. If $(\alpha, \beta)=0$ for any $\beta \in \Sigma_{1}$, then

$$
\left(\widetilde{\lambda}, \alpha^{\vee}\right) \geq 0 .
$$

Proof. First assume $\mathfrak{g}$ is of type $B_{l}$. Let $\alpha \in \Delta\left(\mathfrak{g}, \mathfrak{t}^{c}\right)$. Assume $(\alpha, \beta)=0$ for any $\beta \in \Sigma_{1}$. If $\alpha=\alpha_{i}+\alpha_{i+1}+\cdots+\alpha_{k}$, then $\alpha_{i} \notin \Sigma_{1}$. Similar to the proof of type $A I I I$, one can get $\left(\widetilde{\lambda}, \alpha^{\vee}\right) \geq 0$.

Now we assume $\alpha=\alpha_{i}+\cdots+\alpha_{k-1}+2 \alpha_{k}+\cdots+2 \alpha_{l}$, so $\alpha_{k} \notin \Sigma_{1}$. If $\alpha_{l} \notin \Sigma_{1}$, then we have

$$
\left(\widetilde{\lambda},\left(\alpha_{i}+\cdots+\alpha_{l}\right)^{\vee}\right) \geq 0
$$

and

$$
\left(\widetilde{\lambda},\left(\alpha_{k}+\cdots+\alpha_{l}\right)^{\vee}\right) \geq 0
$$

by Lemmas 3.5 and 3.6. Hence $\left(\widetilde{\lambda}, \alpha^{\vee}\right) \geq 0$.

If $\alpha_{i} \notin \Sigma_{1}$, the proof is similar. So we just need to check the case that $\alpha_{i}$, $\alpha_{l} \in \Sigma_{1}$. Obviously $i+1=k<l$ and $\alpha_{l-1} \notin \Sigma_{1}$. We have

$$
\left(\widetilde{\lambda}, \alpha^{\vee}\right)=\left(\widetilde{\lambda},\left(\alpha_{i}+\cdots+\alpha_{l-1}\right)^{\vee}+\alpha_{l}^{\vee}\right) \geq 0 .
$$

This completes the proof for type $B_{l}$. And the proof for type $C_{l}$ is similar.

This Proposition tells us that those $\beta_{i}$, which satisfy $\left(\widetilde{\lambda}, \beta_{i}^{\vee}\right)<0$, can be chosen to be simple, that is, $\Gamma_{1}=\Sigma_{1}$. Then we get the element $\lambda=$ $\widetilde{\lambda}+\frac{1}{2} \sum_{\beta_{i} \in \Gamma_{1}} \beta_{i}$.

Lemma 3.13. The simple roots of $\mathfrak{l}$ are noncompact simple roots of $\Delta\left(\mathfrak{g}, \mathfrak{t}^{c}\right)$.

Proof. If $\alpha$ is compact simple, then

$$
\left(\lambda, \alpha^{\vee}\right)=\left(\tilde{\lambda}, \alpha^{\vee}\right)+\frac{1}{2} \sum c_{i}\left(\beta_{i}, \alpha^{\vee}\right) \geq 2-\frac{3}{2}>0 .
$$

The first inequality follows from that $\alpha$ is adjacent to at most three simple roots of the same length or two simple roots. So $\alpha \notin \Pi_{\mathfrak{l}}$. 
$\alpha_{1}, \ldots, \alpha_{l-1}$ generate a subsystem of type $A_{l-1}$. Let $\Pi_{\mathfrak{l}}^{\prime}=\Delta\left(\mathfrak{l}, \mathfrak{t}^{c}\right) \cap$ $\left\{\alpha_{1}, \ldots, \alpha_{l-1}\right\}$. Then we have:

Lemma 3.14. Let $\beta_{j} \in \Pi_{\mathfrak{l}}^{\prime} \cap \Sigma_{1}$ and $\alpha \in \Pi_{\mathfrak{l}}^{\prime}$. Then $\left(\beta_{j}, \alpha^{\vee}\right)=0$.

Proof. If $\alpha$ is adjacent to $\beta$, then $\alpha+\beta$ is compact and we have

$$
\begin{aligned}
\left(\lambda,\left(\alpha+\beta_{j}\right)^{\vee}\right) & =\left(\widetilde{\lambda},\left(\alpha+\beta_{j}\right)^{\vee}\right)+\frac{1}{2} \sum c_{i}\left(\beta_{i}, \beta_{j}^{\vee}\right)+\frac{1}{2} \sum c_{i}\left(\beta_{i}, \alpha^{\vee}\right) \\
& \geq 1+c_{j}-\frac{1}{2} c_{j}-1=\frac{1}{2} .
\end{aligned}
$$

This leads to a contradiction.

Corollary 3.15. The Dynkin diagram of $\Pi_{\mathfrak{l}}^{\prime}$ is discrete.

Proof. If it is not true, then there exist two adjacent noncompact simple roots $\alpha, \beta \in \Pi_{\mathfrak{r}}^{\prime}$. By the above Lemma, neither of them is adjacent to some $\beta_{i} \in \Pi_{\mathfrak{l}}^{\prime} \cap \Sigma_{1}$. Then $\alpha+\beta$ is compact.

Case I. $\alpha_{l} \notin \Sigma_{1}$.

$$
\left(\lambda,(\alpha+\beta)^{\vee}\right)=\left(\widetilde{\lambda},(\alpha+\beta)^{\vee}\right) \geq 1+2-2=1 .
$$

Case II. $\alpha_{l} \in \Sigma_{1}$.

1) If $\mathfrak{g}$ is of type $C_{l}$, then $\alpha_{l-1} \notin \Pi_{\mathfrak{l}}$ by the following Lemma 3.16. The inequality (2) is also correct.

2) If $\mathfrak{g}$ is of type $B_{l}$, then

$$
\left(\lambda,(\alpha+\beta)^{\vee}\right) \geq\left(\widetilde{\lambda},(\alpha+\beta)^{\vee}\right)+\frac{1}{2}\left(\alpha_{l},(\alpha+\beta)^{\vee}\right) \geq 1+2-2-\frac{1}{2}=\frac{1}{2} .
$$

Thus for all the cases, we have $\left(\lambda,(\alpha+\beta)^{\vee}\right)>0$. Contradiction.

$\alpha_{l-1}$ and $\alpha_{l}$ generate a subsystem of type $B_{2}=\langle\alpha, \beta\rangle$, where $\alpha$ is the long root.

Lemma 3.16. Let the notation be as above.

1) If $\alpha \in \Sigma_{1}$, then $\left(\lambda, \beta^{\vee}\right)>0$, i.e., $\beta \notin \Pi_{\mathfrak{l}}$.

2) If $\beta \in \Sigma_{1}$, then $\left(\lambda, \alpha^{\vee}\right)>0$, i.e., $\beta \notin \Pi_{\mathfrak{l}}$.

Proof. Thanks to Lemma 3.13, we can assume that $\beta$ is noncompact. Then $\alpha+\beta$ is compact and

$$
\left(\widetilde{\lambda},(\alpha+\beta)^{\vee}\right) \geq 1+2-\left(\rho, 2 \alpha^{\vee}+\beta^{\vee}\right)=0 .
$$

1) $\alpha \in \Sigma_{1}$. Then $\left(\widetilde{\lambda}, \beta^{\vee}\right) \geq 2 c$. For type $B_{l}$,

$$
\left(\lambda, \beta^{\vee}\right) \geq 2+\frac{1}{2}\left(\alpha, \beta^{\vee}\right)=1 \text {. }
$$

For type $C_{l}$,

$$
\left(\lambda, \beta^{\vee}\right) \geq 2+\frac{1}{2}\left(\alpha, \beta^{\vee}\right)+\frac{1}{2}\left(\alpha_{l-2}, \beta^{\vee}\right)=\frac{1}{2}
$$


2) $\beta \in \Sigma_{1}$. Then $\left(\widetilde{\lambda}, \beta^{\vee}\right)=-1$. Since

$$
\left(\widetilde{\lambda},(\alpha+\beta)^{\vee}\right) \geq 0,
$$

then

$$
\left(\widetilde{\lambda}, \alpha^{\vee}\right) \geq \frac{1}{2}
$$

Consequently

$$
\left(\widetilde{\lambda}, \alpha^{\vee}\right) \geq 1
$$

since $\left(\widetilde{\lambda}, \alpha^{\vee}\right)$ is an integer. Then

$$
\left(\lambda, \alpha^{\vee}\right)=\left(\tilde{\lambda}, \alpha^{\vee}\right)+\frac{1}{2}\left(\beta, \alpha^{\vee}\right) \geq \frac{1}{2},
$$

namely, $\alpha \notin \pi_{\text {r }}$.

If $\alpha_{l-1}, \alpha_{l} \notin \Sigma_{1}$, that is,

$$
\left(\tilde{\lambda}, \alpha_{l-1}^{\vee}\right) \geq 0, \quad\left(\tilde{\lambda}, \alpha_{l}^{\vee}\right) \geq 0
$$

then

$$
\left(\widetilde{\lambda},\left(\alpha_{l-1}+\alpha_{l}\right)^{\vee}\right)=2\left(\widetilde{\lambda}, \alpha_{l-1}^{\vee}\right)+\left(\widetilde{\lambda}, \alpha_{l}^{\vee}\right) \geq 0 .
$$

Here the equality holds if and only if

$$
\left(\widetilde{\lambda}, \alpha_{l-1}^{\vee}\right)=\left(\widetilde{\lambda}, \alpha_{l}^{\vee}\right)=0 .
$$

Now we assume $\mathfrak{g}$ is of type $B_{l}$. First we prove a lemma.

Lemma 3.17. Assume $\mathfrak{g}$ is of type $B_{l}$. If $\alpha_{l}$ is noncompact, then $\left(\rho_{c}, \alpha_{l}\right)=$ 0 .

Proof. The compact root $\alpha$ which is adjacent to $\alpha_{l}$ must have one of the two forms: 1) $\left.\alpha=\alpha_{i}+\cdots+\alpha_{l-1}, 2\right) \alpha=\alpha_{i}+\cdots+\alpha_{l-1}+2 \alpha_{l}$. Two such forms occur in a pair. A simple calculation leads to the lemma.

If $\alpha_{l-1} \notin \Sigma_{1}$ and $\mu$ is regular for $\Delta\left(\mathfrak{g}, \mathfrak{t}^{c}\right)$, that is, $(\mu, \alpha) \neq 0$ for any $\alpha \in \Delta\left(\mathfrak{g}, \mathfrak{t}^{c}\right)$, then $\left(\mu+2 \rho_{c}, \alpha_{l}^{\vee}\right) \geq 1$ since $\left(\mu+2 \rho_{c}, \alpha_{l}^{\vee}\right)$ is an integer. Then $\alpha_{l} \notin \Sigma_{1}$. If $\alpha_{l-1}, \alpha_{l} \in \Pi_{\mathfrak{l}}$, then we have $\left(\widetilde{\lambda}, \alpha_{l-1}^{\vee}\right)=\left(\widetilde{\lambda}, \alpha_{l}^{\vee}\right)=0$, that is, $\left(\mu+2 \rho_{c}, \alpha_{l-1}^{\vee}\right)=\left(\mu+2 \rho_{c}, \alpha_{l}^{\vee}\right)=1$. Then $\left(\mu+2 \rho_{c},\left(\alpha_{l-1}+\alpha_{l}\right)^{\vee}\right)=$ $\left(\mu,\left(\alpha_{l-1}+\alpha_{l}\right)^{\vee}\right)+\left(2 \rho_{c},\left(\alpha_{l-1}+\alpha_{l}\right)^{\vee}\right)=\left(\mu,\left(\alpha_{l-1}+\alpha_{l}\right)^{\vee}\right)+2=3$. So we get $\left(\mu, \alpha_{l-1}^{\vee}\right)=0$, which contradicts the assumption that $\mu$ is regular. Actually, we have proved:

Theorem 3.18. Assume $\mathfrak{g}$ is of type $B_{l}$ and $\mu$ is regular for $\Delta\left(\mathfrak{g}, \mathfrak{t}^{c}\right)$. Then the Dynkin diagram of $\mathfrak{l}$ is discrete. Consequently,

$$
\Gamma=\Pi_{\mathfrak{l}} .
$$


3.2.3. Type $D_{l}$. Since all roots of $D_{l}$ have the same length, some results on AIII can be applied and we can get some similar results.

Proposition 3.19. Assume $\mu$ is regular for $\Delta\left(\mathfrak{k}, \mathfrak{t}^{c}\right)$.

1) Let $\alpha \in \Delta^{+}\left(\mathfrak{g}, \mathfrak{t}^{c}\right)$. If $(\alpha, \beta)=0$ for any $\beta \in \Sigma_{1}$, then

$$
\left(\widetilde{\lambda}, \alpha^{\vee}\right) \geq 0 .
$$

2) $\Pi_{\mathfrak{l}}=\Gamma$. Consequently, $\Gamma$ consists of simple roots.

Proof. 1) Let $\left\{\alpha_{1}, \ldots, \alpha_{l}\right\}$ be the simple roots. If $\alpha=\alpha_{i}+\alpha_{i+1}+\cdots+\alpha_{k}$, similar to the proof of Proposition 3.10, one can easily get $\left(\widetilde{\lambda}, \alpha^{\vee}\right) \geq 0$. Now we assume $\alpha=\alpha_{i}+\cdots+\alpha_{k-1}+2 \alpha_{k}+\cdots+2 \alpha_{l-2}+\alpha_{l-1}+\alpha_{l}$.

If $k>i+1$, then $\alpha_{i}, \alpha_{k} \notin \Sigma_{1}$. So we have

$$
\left(\widetilde{\lambda},\left(\alpha_{i}+\cdots+\alpha_{l-1}\right)^{\vee}\right) \geq 0
$$

and

$$
\left(\widetilde{\lambda},\left(\alpha_{k}+\cdots+\alpha_{l-2}+\alpha_{l}\right)^{\vee}\right) \geq 0 .
$$

Hence $\left(\widetilde{\lambda}, \alpha^{\vee}\right) \geq 0$.

If $k=i+1$, that is, $\alpha=\alpha_{i}+2 \alpha_{i+1}+\cdots+2 \alpha_{l-2}+\alpha_{l-1}+\alpha_{l}$, then we have $\alpha_{i+1} \notin \Sigma_{1}$. In this case we may have $\alpha_{i} \in \Sigma_{1}$. If $\alpha_{l-1} \notin \Sigma_{1}$ or $\alpha_{l} \notin \Sigma_{1}$, the proof is similar to the above. Now we assume $\alpha_{l-1}, \alpha_{l} \in \Sigma_{1}$, then $\alpha_{l-2} \notin \Sigma_{1}$. If $\alpha_{l-3} \notin \Sigma_{1}$, then we write

$$
\alpha=\left(\alpha_{i}+\cdots+\alpha_{l-3}\right)+\left(\alpha_{i+1}+\cdots+\alpha_{l-1}\right)+\left(\alpha_{l-2}+\alpha_{l}\right) .
$$

If $\alpha_{l-3} \in \Sigma_{1}$, then $\alpha_{l-4} \notin \Sigma_{1}$, then write

$$
\alpha=\left(\alpha_{i}+\cdots+\alpha_{l-4}\right)+\left(\alpha_{i+1}+\cdots+\alpha_{l-3}\right)+\left(\alpha_{l-3}+2 \alpha_{l-2}+\alpha_{l-1}+\alpha_{l}\right) .
$$

So we need only to show

$$
\left(\widetilde{\lambda},\left(\alpha_{l-3}+2 \alpha_{l-2}+\alpha_{l-1}+\alpha_{l}\right)^{\vee}\right) \geq 0,
$$

where $\alpha_{l-3}, \alpha_{l-1}, \alpha_{l} \in \Sigma_{1}$. If $\alpha_{l-2}$ is compact, then $\left(\widetilde{\lambda}, \alpha_{l-2}^{\vee}\right) \geq 2$. (3) holds. If $\alpha_{l-2}$ is noncompact, then $\alpha_{l-3}+\alpha_{l-2}$ and $\alpha_{l-1}+\alpha_{l-2}$ are compact, hence

$$
\left(\widetilde{\lambda}, \alpha^{\vee}\right)=\left(\widetilde{\lambda},\left(\alpha_{l-3}+\alpha_{l-2}\right)^{\vee}+\left(\alpha_{l-1}+\alpha_{l-2}\right)^{\vee}+\alpha_{l}^{\vee}\right) \geq 1 .
$$

(3) holds.

2) Let $\alpha \in \Pi_{\mathfrak{l}}$. If $\alpha$ is adjacent to $\beta_{j} \in \Sigma_{1}$, then $\alpha+\beta_{j}$ is compact. Then

$$
\begin{aligned}
\left(\lambda,(\alpha+\beta)^{\vee}\right) & =\left(\widetilde{\lambda},(\alpha+\beta)^{\vee}\right)+\frac{1}{2} \sum c_{i}\left(\beta_{i}, \beta^{\vee}\right)+\frac{1}{2} \sum c_{i}\left(\beta_{i}, \alpha^{\vee}\right) \\
& \geq 1+c_{j}-\frac{1}{2} c_{j}-1=\frac{1}{2} .
\end{aligned}
$$

For the first inequality, we use the assumption that $\mu$ is regular. But it contradicts the fact that $\alpha \in \Pi_{\mathfrak{r}}$. 
Now let $\alpha, \beta \in \Pi_{\mathfrak{l}}$ be adjacent. Then neither $\alpha$ nor $\beta$ is adjacent to elements in $\Sigma_{1}$. Again the fact that $\alpha+\beta$ is compact implies it is impossible. So the Dynkin diagram of $\mathfrak{l}$ is discrete. We must have

$$
\Pi_{\mathfrak{l}}=\Gamma \text {. }
$$

Combining the above results, Theorem 3.9 follows.

\section{Dirac cohomology of unitary representations with regular lambda-lowest $K$-types.}

In this section, we will consider the simple group $G$ of types $A I I I(S U(p, q))$, $B I(B I I)\left(S O_{0}(p, q), p+q\right.$ odd $), C I I(S p(p, q)), D I\left(S O_{0}(p, q), p\right.$ and $q$ even), $\operatorname{DIII}\left(S O^{*}(2 n)\right)$, that is all the classical groups except $C I(S p(n, \mathbb{R}))$ with $\operatorname{rank} G=\operatorname{rank} K$. Also we will make the following assumption:

Assumption 4.1. $\mu$ is regular for $\Delta\left(\mathfrak{g}, \mathfrak{t}^{c}\right)$.

4.1. The dominance of $\boldsymbol{\mu}-\boldsymbol{\rho}_{\boldsymbol{n}}$. Since we assume $\mu$ is regular for $\Delta\left(\mathfrak{g}, \mathfrak{t}^{c}\right)$, we can choose the following positive root system for $\Delta\left(\mathfrak{g}, \mathfrak{t}^{c}\right): \alpha \in \Delta^{+}\left(\mathfrak{g}, \mathfrak{t}^{c}\right)$ if $\left(\mu+2 \rho_{c}, \alpha^{\vee}\right)>0$ or $\left(\mu+2 \rho_{c}, \alpha^{\vee}\right)=0$ and $\left(\mu, \alpha^{\vee}\right)>0$. Set $\rho_{n}=\rho-\rho_{c}$.

Since $\mu \in \hat{K}$ is a lambda-lowest $K$-type, then the associate fine $L \cap K$ type with respect to $L$ is $\mu^{L}=\mu-2 \rho(\mathfrak{u} \cap \mathfrak{p})$. Since $\Delta^{+}\left(\mathfrak{l}, \mathfrak{t}^{\mathfrak{c}}\right)=\Pi_{\mathfrak{l}}$ consists of noncompact imaginary roots, we have $\Delta^{+}\left(\mathfrak{l}, \mathfrak{t}^{c}\right) \subset \Delta^{+}\left(\mathfrak{p}, \mathfrak{t}^{c}\right)$, hence

$$
\Delta^{+}\left(\mathfrak{p}, \mathfrak{t}^{c}\right)=\Delta^{+}\left(\mathfrak{l}, \mathfrak{t}^{c}\right) \cup \Delta\left(\mathfrak{u}, \mathfrak{t}^{c}\right) .
$$

So $\rho(\mathfrak{u} \cap \mathfrak{p})=\rho_{n}-\rho_{\mathfrak{l}}=\rho-\rho_{c}-\rho_{\mathfrak{l}}$. Consequently,

$$
\begin{aligned}
\mu^{L} & =\mu-2 \rho(\mathfrak{u} \cap \mathfrak{p}) \\
& =\mu+2 \rho_{c}-2\left(\rho-\rho_{\mathfrak{l}}\right) .
\end{aligned}
$$

So

$$
\left(\mu^{L}, \beta_{i}^{\vee}\right)=1-c_{i}
$$

for $\beta_{i} \in \Pi_{\mathfrak{r}}$.

Proposition 4.2. $\mu-\rho_{n}$ is dominant for $\Delta^{+}\left(\mathfrak{k}, \mathfrak{t}^{c}\right)$.

We have to deal with it case by case.

Proof of the case AIII. We just need to prove $\widetilde{\lambda}$ is strictly dominant for $\Delta\left(\mathfrak{k}, \mathfrak{t}^{c}\right)$, that is,

$$
\left(\tilde{\lambda}, \alpha^{\vee}\right) \geq 1
$$

for any $\alpha$ compact. Let $\Pi=\left\{\alpha_{1}, \ldots, \alpha_{l}\right\}$ be the simple roots of $\Delta^{+}\left(\mathfrak{g}, \mathfrak{t}^{c}\right)$. Then the system of simple roots $\Pi_{\mathfrak{k}}$ of $\Delta^{+}\left(\mathfrak{k}, \mathfrak{t}^{c}\right)$ consists of two kinds of elements

$$
\Pi_{\mathfrak{k}}=\Pi_{c} \cup \Pi_{n} .
$$


$\Pi_{c}$ consists of those compact simple roots of $\Pi$. Elements of $\Pi_{n}$ are of the form

$$
\alpha=\alpha_{i}+\cdots+\alpha_{k}
$$

where $\alpha_{i}$ and $\alpha_{k}$ are noncompact and $\alpha_{i+1}, \ldots, \alpha_{k-1}$ are compact. If $\alpha \in \Pi_{c}$ then

$$
\left(\widetilde{\lambda}, \alpha^{\vee}\right) \geq 2
$$

If $\alpha \in \Pi_{n}$, we treat it case by case.

Case I. $\alpha=\alpha_{i}+\alpha_{i+1}$, where $\alpha_{i}, \alpha_{i+1}$ are noncompact. Then

$$
\left(\widetilde{\lambda}, \alpha^{\vee}\right) \geq 1+2-2=1 \text {. }
$$

Case II. $\alpha=\alpha_{i}+\cdots+\alpha_{k}$, where $k-i>2$. In this case $\alpha_{i+1}$ and $\alpha_{i+2}$ are compact. Then

$$
\left(\widetilde{\lambda}, \alpha^{\vee}\right)=\left(\widetilde{\lambda},\left(\alpha_{i}+\alpha_{k}\right)^{\vee}\right)+\left(\widetilde{\lambda},\left(\alpha_{i+1}+\cdots+\alpha_{k-1}\right)^{\vee}\right) \geq 1 .
$$

Case III. $\alpha=\alpha_{i}+\alpha_{i+1}+\alpha_{i+2}$. Here $\alpha_{i+1}$ is compact. Then $\left(\widetilde{\lambda}, \alpha^{\vee}\right) \geq 0$. If $\left(\tilde{\lambda}, \alpha^{\vee}\right)=0$, then

$$
\left(\widetilde{\lambda}, \alpha_{i}\right)=\left(\widetilde{\lambda}, \alpha_{i+2}\right)=-1, \quad\left(\widetilde{\lambda}, \alpha_{i+1}\right)=2 .
$$

Thus both $\left(\mu, \alpha_{i}\right)$ and $\left(\mu, \alpha_{i+2}\right)$ are integers. $\left(\rho_{c}, \alpha_{i}^{\vee}+\alpha_{i+2}^{\vee}\right)=\left(\rho_{c}, \alpha^{\vee}\right)-$ $\left(\rho_{c}, \alpha_{i+1}^{\vee}\right)=0$ implies that $\left(\mu, \alpha_{i}^{\vee}+\alpha_{i+2}^{\vee}\right)=0$, which contradicts the choice of positive roots.

Proof of the case $B_{l}$. Let $\alpha \in \Pi_{\mathfrak{k}}$ be simple. Then $\alpha$ must be one of the forms in the following cases:

Case I. $\alpha=\alpha_{i} \in \Pi$. Then $\left(\mu-\rho_{n}, \alpha^{\vee}\right) \geq 1$.

Case II. $\alpha=\alpha_{i}+\cdots+\alpha_{k}$, where $\alpha_{i}$ and $\alpha_{k}$ are noncompact and others are compact.

If $k<l$, the proof is similar to that of $A I I I$.

If $k=l$, that is, $\alpha_{l}$ is noncompact, then $\left(\widetilde{\lambda}, \alpha^{\vee}\right)=\left(\widetilde{\lambda}, 2\left(\alpha_{i}^{\vee}+\cdots+\alpha_{l-1}^{\vee}\right)+\right.$ $\left.\alpha_{l}^{\vee}\right)$. If $i<l-1$, we can get the result easily. If $i=l-1$, then $\left(\rho_{c}, \alpha_{l}^{\vee}\right)=0$ implies $2\left(\rho_{c}, \alpha_{l-1}^{\vee}\right)=1$ and $\left(\mu, \alpha_{l}^{\vee}\right) \geq 1$. Since $\mu$ is an integral weight and the choice of positive root system depends on $\mu$, we have

$$
\left(\mu, \alpha_{l-1}^{\vee}\right) \geq 0 \text {. }
$$

Since

$$
\left(\mu, \alpha_{l}^{\vee}\right) \geq 0,
$$

and the equalities (4) and (5) can't hold at the same time, we have

$$
\left(\widetilde{\lambda},\left(\alpha_{l-1}+\alpha_{l}\right)^{\vee}\right) \geq 1 \text {. }
$$

Case III. $\alpha=\alpha_{i}+\cdots+\alpha_{k}+2\left(\alpha_{k+1}+\cdots+\alpha_{l}\right)$, where $\alpha_{i}, \alpha_{k}$ and $\alpha_{k+1}$ are noncompact and the others are compact. 
Since $\alpha_{i}+\cdots+\alpha_{k} \in \Pi_{\mathfrak{k}}$, we need only to prove

$$
\left(\widetilde{\lambda},\left(\alpha_{k+1}+\cdots+\alpha_{l}\right)^{\vee}\right) \geq 0,
$$

which is obvious thanks to Lemma 3.17 .

Proof of the case CII. Since $\mathfrak{g}$ is of type $C I I, \mathfrak{k}$ has no center and $\alpha_{l}$ must be a compact root. Let $\alpha \in \Pi_{\mathfrak{k}}$ be simple. Then $\alpha$ must be one of the forms in the following cases:

Case I. Similar to type $B_{l}$.

Case II. $\alpha=\alpha_{i}+\cdots+\alpha_{k}$, where $k<l$. Similar to type $B_{l}$.

Case III. $\alpha=2\left(\alpha_{i}+\cdots+\alpha_{l-1}\right)+\alpha_{l}$, where only $\alpha_{i}$ is noncompact.

Since $\left(\tilde{\lambda}, \alpha^{\vee}\right)=\left(\widetilde{\lambda}, \alpha_{i}^{\vee}+\cdots+\alpha_{l}^{\vee}\right)$, the conclusion is clear.

Proof of the case $D_{l}$. Let $\alpha \in \Pi_{\mathfrak{k}}$ be simple. Then $\alpha$ must be one of the forms in the following cases:

Case I. Similar to type $B_{l}$.

Case II. $\alpha=\alpha_{i}+\cdots+\alpha_{k}(k \leq l-2), \alpha=\alpha_{i}+\cdots+\alpha_{l-1}$ or $\alpha=$ $\alpha_{i}+\cdots+\alpha_{l-2}+\alpha_{l}$ or $\alpha_{l-1}+\alpha_{l-2}+\alpha_{l}$. Still similar to type $B_{l}$.

Case III. $\alpha=\alpha_{i}+\cdots+\alpha_{l}$, where $\alpha_{i}, \alpha_{l-1}, \alpha_{i-1}$ and $\alpha_{l}$ are noncompact and others are compact.

The only hard case is that $i=l-3$. Since at least one of $\alpha_{l-3}, \alpha_{l-1}$ and $\alpha_{l}$ is not in $\Sigma_{1}$, all the simple root of $\mathfrak{k}$ is one of the three forms:

1) $\alpha_{i} \in \Pi, i<l-3$.

2) $\alpha_{i}+\cdots+\alpha_{k}, k<l-2$.

3) $\alpha_{l-3}+\alpha_{l-2}, \alpha_{l-2}+\alpha_{l-1}, \alpha_{l-2}+\alpha_{l}$ or $\alpha_{l-3}+\alpha_{l-2}+\alpha_{l-1}+\alpha_{l}$.

In this case $\mathfrak{k}$ is a sum of two simple Lie algebras of type $D_{l}$, say $\mathfrak{k}=\mathfrak{k}_{1} \oplus \mathfrak{k}_{2}$ and $\Pi_{\mathfrak{k}}=\Pi_{\mathfrak{k}_{1}} \cup \Pi_{\mathfrak{k}_{2}}$. One can easily see $\alpha_{l-3}+\alpha_{l-2}$ and $\alpha_{l-3}+\alpha_{l-2}+\alpha_{l-1}+\alpha_{l}$ belong to the same subsystem, say $\Pi_{\mathfrak{k}_{1}}$, while $\alpha_{l-2}+\alpha_{l-1}, \alpha_{l-2}+\alpha_{l} \in \Pi_{\mathfrak{k}_{2}}$. And they play the role of $\alpha_{l-1}$ and $\alpha_{l}$. One can easily see that

$$
\left(\rho_{c}, \alpha_{l-1}^{\vee}\right)=\left(\rho_{c}, \alpha_{l}^{\vee}\right)=0 .
$$

Then $\left(\mu, \alpha_{l-1}^{\vee}\right)>0$ and $\left(\mu, \alpha_{l}^{\vee}\right)>0$ and $\left(\rho_{c}, \alpha_{l-2}^{\vee}\right)=1$. If $\mu$ is an integral weight, then $\alpha_{l-1}, \alpha_{l} \notin \Sigma_{1}$. The assertion follows.

Case IV. $\alpha=\alpha_{i}+\cdots+\alpha_{k}+2\left(\alpha_{k+1}+\cdots+\alpha_{l-2}\right)+\alpha_{l-1}+\alpha_{l}$, where $\alpha_{i}+\cdots+\alpha_{k}(k \leq l-2)$ is a compact root in case II and $\alpha_{k+1}, \alpha_{l-1}$ and $\alpha_{l}$ are noncompact. We can easily get the $\left(\mu-\rho_{n}, \alpha^{\vee}\right) \leq 0$. 
4.2. The dominance of $\Lambda$. Let $\Lambda=\lambda+\frac{1}{2} \sum c_{i} \beta_{i}=\widetilde{\lambda}+\sum c_{i} \beta_{i}$. Then we have:

Proposition 4.3. $\Lambda$ is dominant for $\Delta^{+}\left(\mathfrak{g}, \mathfrak{t}^{c}\right)$.

Proof. Let $\sigma_{i} \in W\left(\mathfrak{g}, \mathfrak{t}^{c}\right)$ be the reflection with respect to $\beta_{i} \in \Gamma$. Set $\Delta^{\prime}=\left\{\alpha \in \Delta^{+}\left(\mathfrak{g}, \mathfrak{t}^{c}\right) \mid \alpha \notin \Gamma\right\}$. Then $\Delta^{\prime}$ is stable under each $\sigma_{i}$ and their product $\sigma=\sigma_{1} \ldots \sigma_{r}$.

$\Lambda=\sigma(\widetilde{\lambda})$ is dominant for $\Delta^{\prime}$ if and only if $\widetilde{\lambda}$ is dominant for $\Delta^{\prime}$. The assertion follows by the following lemma.

Lemma 4.4. $\tilde{\lambda}$ is dominant for $\Delta^{\prime}$.

Proof. In the above subsection, we have proved that $\widetilde{\lambda}$ is strictly dominant for $\Delta^{+}\left(\mathfrak{k}, \mathfrak{t}^{c}\right)$, so the only left is to check our assertion for noncompact roots in $\Delta^{\prime}$.

Let $\alpha \in \Delta^{\prime}$ be noncompact. If $\alpha$ is not adjacent to any element in $\Sigma_{1}$, then

$$
\left(\widetilde{\lambda}, \alpha^{\vee}\right)=\left(\lambda, \alpha^{\vee}\right)>0 .
$$

Now assume $\alpha$ is adjacent to $\beta \in \Sigma_{1}$. If $\alpha+\beta$ is a root, then it is compact. So we have

$$
\left(\widetilde{\lambda},(\alpha+\beta)^{\vee}\right) \geq 1 \text {. }
$$

One can easily get $\left(\widetilde{\lambda}, \alpha^{\vee}\right)>0$. If $\gamma=\alpha-\beta$ is a root then

$$
\left(\widetilde{\lambda}, \alpha^{\vee}\right)=\left(\widetilde{\lambda},(\gamma+\beta)^{\vee}\right) \text {. }
$$

Also we have $\left(\widetilde{\lambda}, \alpha^{\vee}\right) \geq 0$ if $(\gamma, \gamma) \geq(\beta, \beta)$ or $\gamma$ is not a simple compact root. So we just need to consider the case that $(\gamma, \gamma)<(\beta, \beta)$ and $\gamma$ is a simple compact root. Obviously, $\mathfrak{g}$ is of type $B_{l}$ and $\gamma=\alpha_{i}+\cdots+\alpha_{l}$. According to the proof in Chapter 3, the assertion follows.

4.3. The representations of $\boldsymbol{L}$. Let $L_{1}$ be the commutator subgroup of $L$. It is a connected semisimple Lie group by [8, Lemma 4.3.4]. Then $L=T L_{1}$ (see [8, Lemma 0.4.2]) and $T_{1}=T \cap L_{1}$ is a finite product of $\mathbb{Z}_{2}$. Let $(\delta, V) \in \hat{T}$ and $\delta_{1}=\left.\delta\right|_{T_{1}}$. Then $\left(\delta_{1}, V\right) \in \hat{T}$. Define $(\pi, \mathcal{H})=\pi(P, \delta \otimes \nu)$ and $\left(\pi_{1}, \mathcal{H}_{1}\right)=\pi_{1}\left(P_{1}, \delta \otimes \nu\right)$, where $P=T A N, P_{1}=T_{1} A N$ and $\nu \in \hat{A}$.

Lemma 4.5. $\left.\pi\right|_{L_{1}} \cong \pi_{1}$ as representations of $L_{1}$. Consequently, $\pi$ is irreducible (resp. unitary) if and only if $\pi_{1}$ is irreducible (resp. unitary).

Locally, $L_{1}$ is a product of some copies of $S L(2, \mathbb{R})$, i.e., there exists a canonical covering map:

$$
p: \widetilde{L_{1}}=S L(2, \mathbb{R}) \times \cdots \times S L(2, \mathbb{R}) \rightarrow L_{1}
$$

with finite kernel $Z$. Then $\pi_{1}$ can be regarded as a representation of $\widetilde{L}$ with $Z$ acting trivially. Let $\widetilde{T}=p^{-1}\left(T_{1}\right)$. Then $\delta_{1}$ can be regarded as a 
representation of $\widetilde{T_{1}}$. Let $\widetilde{\pi}=\pi\left(\widetilde{P}, \delta_{1} \otimes \nu\right)$, which is equivalent with $\pi_{1}$ as representations of $\widetilde{L_{1}}$. Obviously, $\pi_{1}$ is a tensor product of representations of $S L(2, \mathbb{R})$. Then $\pi_{1}$ is unitary (irreducible, resp.) if and only if every component of the tensor product is unitary (irreducible, resp.). We can easily get the unitaribility and irreducibiliby of representations of $L_{1}$ since the representations of $S L(2, \mathbb{R})$ is so clear. Let us recall the following:

Theorem 4.6 ([4], Theorem 16.3). The only irreducible unitary representations of $S L(2, \mathbb{R})$ up to unitary equivalence are:

a) The trivial representation;

b) the discrete series $\mathcal{D}_{n}^{ \pm}, n \geq 2$, and the limits of discrete series $\mathcal{D}_{1}^{ \pm}$,

c) the irreducible members of the unitary principal series, $\mathcal{P}^{+, i y}$ with $y$ real and $\mathcal{P}^{-, i y}$ with $y$ nonzero real,

d) the complementary series $\wp^{x}$ with $0<x<1$.

Moreover the only equivalences among these representations are $\mathcal{P}^{+, i y} \cong$ $\mathcal{P}^{+,-i y}$ and $\mathcal{P}^{-, i y} \cong \mathcal{P}^{-,-i y}$.

The fine representation $\mu^{0}$ (see [8], Corollary 5.4.7) corresponding to $\mu$ is just $\mu^{0}=\mu-2 \rho(\mathfrak{u} \cap \mathfrak{p})=\mu-2 \rho(\mathfrak{p})+2 \rho(\mathfrak{l})=\left(\mu+2 \rho_{c}\right)-2(\rho-\rho(\mathfrak{l}))$. Then we have

$$
\left(\mu^{0}, \beta_{i}^{\vee}\right)=1-c_{i},
$$

that is, $\mu^{0}$ is weight 0 of those $\mathfrak{l}\left(\beta_{i}\right)$ for $\beta_{i} \in \Gamma_{1}$ (Here $\mathfrak{l}\left(\beta_{i}\right)$ is the TDS generated by $\left.\beta_{i}\right)$ and weight 1 of those $\mathfrak{l}\left(\beta_{i}\right)$ for $\beta_{i} \in \Gamma_{0}$. Consequently $L\left(\beta_{i}\right) \cong S L(2, \mathbb{R})$ since $L\left(\beta_{i}\right)$ is either $S L(2, \mathbb{R})$ or $P S L(2, \mathbb{R})$, but the representations of the latter has no odd weight.

4.4. Proof of Theorem 1.2. Let $X$ be an irreducible $(\mathfrak{g}, K)$-module with lambda-lowest $K$-type $\mu$ satisfying $\mu$ is regular for $\Delta\left(\mathfrak{g}, \mathfrak{t}^{c}\right)$. By the discussion above, we have known the following facts:

1) $\lambda=\mu+2 \rho_{c}-\rho+\frac{1}{2} \sum_{\beta_{i} \in \Gamma_{1}} \beta_{i}$. Let $\mathfrak{q}=\mathfrak{l}+\mathfrak{u}$ be the parabolic associated to $\mu$. Then the Dynkin diagram of $\mathfrak{l}$ is discrete.

2) $\mu-\rho_{n}$ is dominant for $\Delta^{+}\left(\mathfrak{k}, \mathfrak{t}^{c}\right)$.

3) $\Lambda=\left(\lambda, \frac{1}{2} \sum_{\beta_{i} \in \Gamma_{1}} \beta_{i}\right)$ is dominant for $\Delta^{+}\left(\mathfrak{g}, \mathfrak{t}^{c}\right)$.

Now we assume the $\theta$-stable data corresponding to $X$ is $(\mathfrak{q}, H, \delta, \nu)$, where $\nu=\frac{1}{2} \sum_{\beta_{i} \in \Gamma_{1}} \beta_{i}$. Consider the standard $(\mathfrak{g}, K)$-module:

$$
\mathcal{R}^{S}\left(X_{L}(P, \delta \otimes \nu)\right)
$$

By Theorem 6.5.12 [8], $X \cong \mathcal{R}^{S}\left(X_{L}(P, \delta \otimes \nu)\right)(\mu)$ and a canonical cohomology class is $Y=\bar{X}_{L}(P, \delta \otimes \nu)(\mu-2 \rho(\mathfrak{u} \cap \mathfrak{p}))$, which is unitary as one can easily see. $\mathcal{R}^{S}(Y)$ is a submodule of $\mathcal{R}^{S}\left(X_{L}(P, \delta \otimes \nu)\right)$. We have $X \subseteq \mathcal{R}^{S}(Y)$ since they have the same lambda-lowest $K$-type $\mu$. Since $\left(\Lambda, \alpha^{\vee}\right) \geq 0$, then 
$\mathcal{R}^{S}(Y)$ is unitary and irreducible (it is nonzero since it contains $X$ ), hence $X=\mathcal{R}^{S}(Y)$ is unitary. By Dirac inequality, we have

$$
\left(\omega\left(\mu^{\prime}-\rho_{n}^{\prime}\right)+\rho_{c}, \omega\left(\mu^{\prime}-\rho_{n}^{\prime}\right)+\rho_{c}\right) \geq(\Lambda, \Lambda),
$$

for all $K$-type $\mu^{\prime}$ of $X$, all $\rho_{n}^{\prime}$ and for some $\omega \in W_{K}$. Note that $\mu-\rho_{n}$ is dominant for $\Delta^{+}\left(\mathfrak{k}, \mathfrak{t}^{c}\right)$ and

$$
\mu-\rho_{n}+\rho_{c}=\Lambda
$$

we have the equality holds. Using $t \nu, 0<t<1$, instead of $\nu$, one can see that $X_{L}(\delta \otimes t \nu)$ is unitary since it is a tensor product of complementary series and discrete series of $S L(2, \mathbb{R})$. Let $\Lambda_{t}=(\lambda, t \nu)$. Then $\Lambda_{0}=\lambda$ and $\Lambda_{t}=(1-t) \Lambda_{0}+t \Lambda_{1}$. Hence $\left(\Lambda_{t}, \alpha^{\vee}\right)=(1-t)\left(\lambda, \alpha^{\vee}\right)+t\left(\widetilde{\lambda}, \alpha^{\vee}\right)>0$, for all $\alpha \in \Delta(\mathfrak{u})$. Then by Theorem $1.3[\mathbf{9}]$, we have

$$
\mathcal{R}^{S}\left(X_{L}(\delta \otimes t \nu)\right)
$$

is unitary. So we have

$$
\left(\omega\left(\mu^{\prime}-\rho_{n}^{\prime}\right)+\rho_{c}, \omega\left(\mu^{\prime}-\rho_{n}^{\prime}\right)+\rho_{c}\right) \geq\left(\Lambda_{t}, \Lambda_{t}\right),
$$

for all $t \in(0,1)$ by Dirac inequality. Since all the $K$ types of $X_{G}(\mathfrak{q}, H, \delta, \nu)$ are independent of the choice of $\nu$, when $t$ tends to 1 , we get

$$
\left(\omega\left(\mu^{\prime}-\rho_{n}^{\prime}\right)+\rho_{c}, \omega\left(\mu^{\prime}-\rho_{n}^{\prime}\right)+\rho_{c}\right) \geq(\Lambda, \Lambda)
$$

which implies $\left(\mu-\rho_{n}+\rho_{c}, \mu-\rho_{n}+\rho_{c}\right)=(\Lambda, \Lambda)$, hence $X$ has nonzero Dirac cohomology.

Conversely, if $X$ has nonzero Dirac cohomology, then the infinitesimal character of $X$ is $\mu-\rho_{n}+\rho_{c}=(\lambda, \nu)$ by the same argument. One can easily show that $\nu=\frac{1}{2} \sum_{\beta_{i} \in \Gamma_{1}} \beta_{i}$.

\section{References}

[1] S. Helgason, Differential Geometry, Lie Groups and Symmetric Spaces, Academic Press, New York, 1978, MR 0514561, Zbl 0451.53038.

[2] J. Huang and P. Pandžić, Dirac cohomology, unitary representations and a proof of a Conjecture of Vogan, J. Amer. Math. Soc., 15 (2002), 185-202, MR 1862801, Zbl 0980.22013 .

[3] J.E. Humphreys, Introduction to Lie Algebras and Representation Theory, SpringerVerlag, Berlin-Heildelberg-New York, 1972, MR 0323842, Zbl 0254.17004.

[4] A. Knapp, Representation Theory of Semisimple Groups: An Overview Based on Examples, Princeton University Press, Princeton, 1986, MR 1880691, Zbl 0604.22001.

[5] A.W. Knapp and D. Vogan, Cohomological Induction and Unitary Representations, Princeton University Press, Princeton, 1995, MR 1330919, Zbl 0863.22011.

[6] P. Parthasarathy, The Dirac operator and the discrete series, Ann. of Math., 96 (1972), 1-30, MR 0318398, Zbl 0249.22003. 
[7] S.A. Salamanca-Riba, On the unitary dual of real reductive Lie groups and the $A_{\mathfrak{q}}(\lambda)$ modules: The strongly regular case, Duke Math. J., 96 (1999), 521-546, MR 1671213, Zbl 0941.22014.

[8] D.A. Vogan, Jr., Representations of Real Reductive Lie Groups, Birkhäuser Boston, 1981, MR 0632407, Zbl 0469.22012.

[9] _ Unitarizability of certain series of representations, Ann. of Math., 120 (1984), 141-187, MR 0750719, Zbl 0561.22010.

[10] D.A. Vogan, Jr. and G. Zuckerman, Unitary representations with nonzero cohomology, Compositio Math., 53 (1984), 51-90, MR 0762307, Zbl 0692.22008.

[11] N. Wallach, Real Reductive Groups I, Academic Press, San Diego, 1988, MR 0929683, Zbl 0666.22002.

Received February, 12, 2003. This work is supported by the National Science Foundation of China (10171048, 10271058).

College of Mathematical Sciences

NANKAI UNIVERSITY

TiAnJin 300071

China

E-mail address: houzx@nankai.edu.cn

College of Mathematical Sciences and LPMC

NANKAI UNIVERSITY

TiANJIN 300071

ChInA

E-mail address: liangke@nankai.edu.cn

School of Mathematical Sciences

PEKING University

BEIJING 100871

CHina

E-mail address: zhufuhai@math.pku.edu.cn 